\title{
COVID-19 infection: the perspectives on immune responses
}

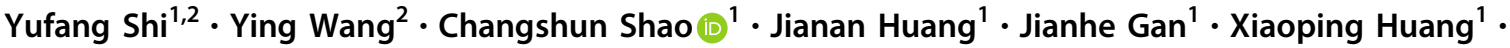 \\ Enrico Bucci $\circledast^{3,4}$ - Mauro Piacentini ${ }^{5}$ - Giuseppe Ippolito $^{5}$ • Gerry Melino ${ }^{6,7}$
}

Received: 1 March 2020 / Revised: 10 March 2020 / Accepted: 10 March 2020 / Published online: 23 March 2020

(c) ADMC Associazione Differenziamento e Morte Cellulare 2020

More than 100 years since the outbreak of the 1918 influenza pandemic, we now seem to face another pandemic. The outbreak of the new coronavirus (SARS-CoV-2) infection is spreading to every continent, forcing us to live with this virus for perhaps a long time. Scientists and clinicians have learned much of coronavirus disease 2019, COVID-19, and its pathogenesis [1]: not all people exposed to SARS-CoV-2 are infected and not all infected patients develop severe respiratory illness. Accordingly, SARSCoV-2 infection can be roughly divided into three stages: stage I, an asymptomatic incubation period with or without detectable virus; stage II, non-severe symptomatic period with the presence of virus; stage III, severe respiratory symptomatic stage with high viral load [2]. From the point of view of prevention, individuals at stage $\mathrm{I}$, the stealth carriers, are the least manageable because, at least on some occasions, they spread the virus unknowingly: indeed, the first asymptomatic transmission has been reported in Germany [3]. The role of asymptomatic SARS-CoV-2 infected

Yufang Shi

yfshi@suda.edu.cn

1 The First Affiliated Hospital of Soochow University, State Key Laboratory of Radiation Medicine and Protection, Institutes for Translational Medicine, Soochow University Medical College, Suzhou, China

2 Shanghai Institute of Nutrition and Health, Shanghai Institutes for Biological Sciences, Chinese Academy of Sciences, 320 Yueyang Road, Shanghai 200031, China

3 Sbarro Health Research Organization, Temple University, Philadelphia, PA 19122, USA

4 Resis Srl, 10010 Samone, TO, Italy

5 National Institute for Infectious Diseases 'Lazzaro Spallanzani” IRCCS, 00149 Rome, Italy

6 Department of Experimental Medicine, TOR, University of Rome Tor Vergata, 00133 Rome, Italy

7 Medical Research Council (MRC) Toxicology Unit, University of Cambridge, Cambridge CB2 1QP, UK individuals in disseminating the infection remains to be defined.

Among over 1000 patients analyzed in Wuhan, except occasionally in children and adolescence, it infects all the other age groups evenly. About $15 \%$ of the confirmed cases progress to the severe phase, although there is a higher chance for patients over 65 to progress into the severe phase [1]. One of the biggest unanswered questions is why some develop severe disease, whilst others do not. Clearly, the conventional wisdom based on overall immunity of the infected patients cannot explain this broad spectrum in disease presentation.

\section{Two-phase immune responses induced by COVID-19 infection}

Clinically, the immune responses induced by SARS-CoV-2 infection are two phased. During the incubation and nonsevere stages, a specific adaptive immune response is required to eliminate the virus and to preclude disease progression to severe stages. Therefore, strategies to boost immune responses (anti-sera or pegylated IFN $\alpha$ ) at this stage are certainly important. For the development of an endogenous protective immune response at the incubation and non-severe stages, the host should be in good general health and an appropriate genetic background (e.g. HLA) that elicits specific antiviral immunity. Genetic differences are well-known to contribute to individual variations in the immune response to pathogens. However, when a protective immune response is impaired, virus will propagate and massive destruction of the affected tissues will occur, especially in organs that have high ACE2 expression, such as intestine and kidney. The damaged cells induce innate inflammation in the lungs that is largely mediated by proinflammatory macrophages and granulocytes. Lung inflammation is the main cause of life-threatening respiratory disorders at the severe stage [4]. Therefore, good general health may not be advantageous for patients who have advanced to the severe stage: once severe lung damage 
occurs, efforts should be made to suppress inflammation and to manage the symptoms.

Alarmingly, after discharge from hospital, some patients remain/return viral positive and others even relapse. This indicates that a virus-eliminating immune response to SARS-CoV-2 may be difficult to induce at least in some patients and vaccines may not work in these individuals. Those recovered from the non-severe stage should be monitored for the virus together with T/B cell responses. These scenarios should be considered when determining the strategies of vaccine development. In addition, there are many types or subtypes of coronavirus. Thus, if vaccines directly targeting SARS-CoV-2 prove to be difficult to develop, the Edward Jenner approach should be considered.

\section{Cytokine storm and lung damage}

The cytokine release syndrome (CRS) seems to affect patients with severe conditions. Since lymphocytopenia is often seen in severe COVID-19 patients, the CRS caused by SARS-CoV-2 virus has to be mediated by leukocytes other than $\mathrm{T}$ cells, as in patients receiving CAR-T therapy; a high WBC-count is common, suggesting it, in association with lymphocytopenia, as a differential diagnostic criterion for COVID-19. In any case, blocking IL-6 may be effective. Blocking IL-1 and TNF may also benefit patients. Although various clinical sites in China have announced the use of mesenchymal stromal/stem cells (MSCs) in severe cases with COVID-19 infection, solid results have yet to be seen. One caveat is that MSCs need to be activated by IFN $\gamma$ to exert their anti-inflammatory effects, which may be absent in severely affected patients as $\mathrm{T}$ cells are not well activated by SARS-CoV-2 infection. To enhance effectiveness, one could consider employing the "licensingapproach": pretreat MSCs with IFN $\gamma$ with/without TNF or IL1 [5]. Such cytokine-licensed MSCs could be more effective in the suppression of hyperactive immune response and promotion of tissue repair, as licensed-MSCs are effective in LPSinduced acute lung damage [6].

Lung damage is a major hurdle to recovery in those severe patients. Through producing various growth factors, MSCs may help repair of the damaged lung tissue. It is important to mention that various studies have shown that in animal models with bleomycin-induced lung injury, vitamin B3 (niacin or nicotinamide) is highly effective in preventing lung tissue damage [7]. It might be a wise approach to supply this food supplement to the COVID-19 patients.

\section{HLA haplotypes and SARS-CoV-2 infection}

The major-histocompatibility-complex antigen loci (HLA) are the prototypical candidates for genetic susceptibility to infectious diseases [8, 9]. Haplotype HLA-loci variability results from selective pressure during co-evolution with pathogens. Immunologists have found that T-cell antigen receptors, on $\mathrm{CD}^{+}{ }^{+}$or $\mathrm{CD}^{+} \mathrm{T}$ cells recognize the conformational structure of the antigen-binding-grove together with the associated antigen peptides. Therefore, different HLA haplotypes are associated with distinct disease susceptibilities. The repertoire of the HLA molecules composing a haplotype determines the survival during evolution. Accordingly, it seems advantageous to have HLA molecules with increased binding specificities to the SARS-CoV-2 virus peptides on the cell surface of antigenpresenting cells. Indeed, the susceptibility to various infectious diseases such as tuberculosis, leprosy, HIV, hepatitis B, and influenza is associated with specific HLA haplotypes. Particular murine MHC class II haplotypes are associated with the susceptibility to influenza. In man, HLA class $I$ is also associated with H1N1 infections: HLA-A*11, HLA-B $* 35$, and HLA-DRB $1 * 10$ confers susceptibility to influenza $A(H 1 N 1) p d m 09$ infection [10]. Therefore, it is imperative to study whether specific HLA loci are associated with the development of anti-SARS-CoV-2 immunity and, if so, to identify the alleles, either class I or II, that demonstrate induction of protective immunity. Once the dominant alleles are identified, simple detection kits can be developed. Such information is critical for (1) strategic clinical management; (2) evaluation of the efficacy of vaccination in different individuals in the general population; (3) assignment of clinical professional and managerial teams amid interactions with COVID-19 patients.

\section{Hyaluronan: a potential cause of fatalities}

The innate immune response to tissue damage caused by the virus could lead to acute respiratory distress syndrome (ARDS), in which respiratory failure is characterized by the rapid onset of widespread inflammation in the lungs and subsequent fatality [4]. The symptoms of ARDS patients include short/rapid breathing, and cyanosis. Severe patients admitted to intensive care units often require mechanical ventilators and those unable to breath have to be connected to extracorporeal membrane oxygenation (ECMO) to support life [11]. CT images revealed that there are characteristic white patches called "ground glass", containing fluid in the lungs [2]. Recent autopsies have confirmed that the lungs are filled with clear liquid jelly, much resembling the lungs of wet drowning [4]. Although the nature of the clear jelly has yet to be determined, hyaluronan (HA) is associated with ARDS [12]; moreover, during SARS infection, the production and regulation of hyaluronan is defective. The levels of inflammatory cytokines (IL-1, TNF) are high in the lungs of COVID-19 patients and these cytokines are 


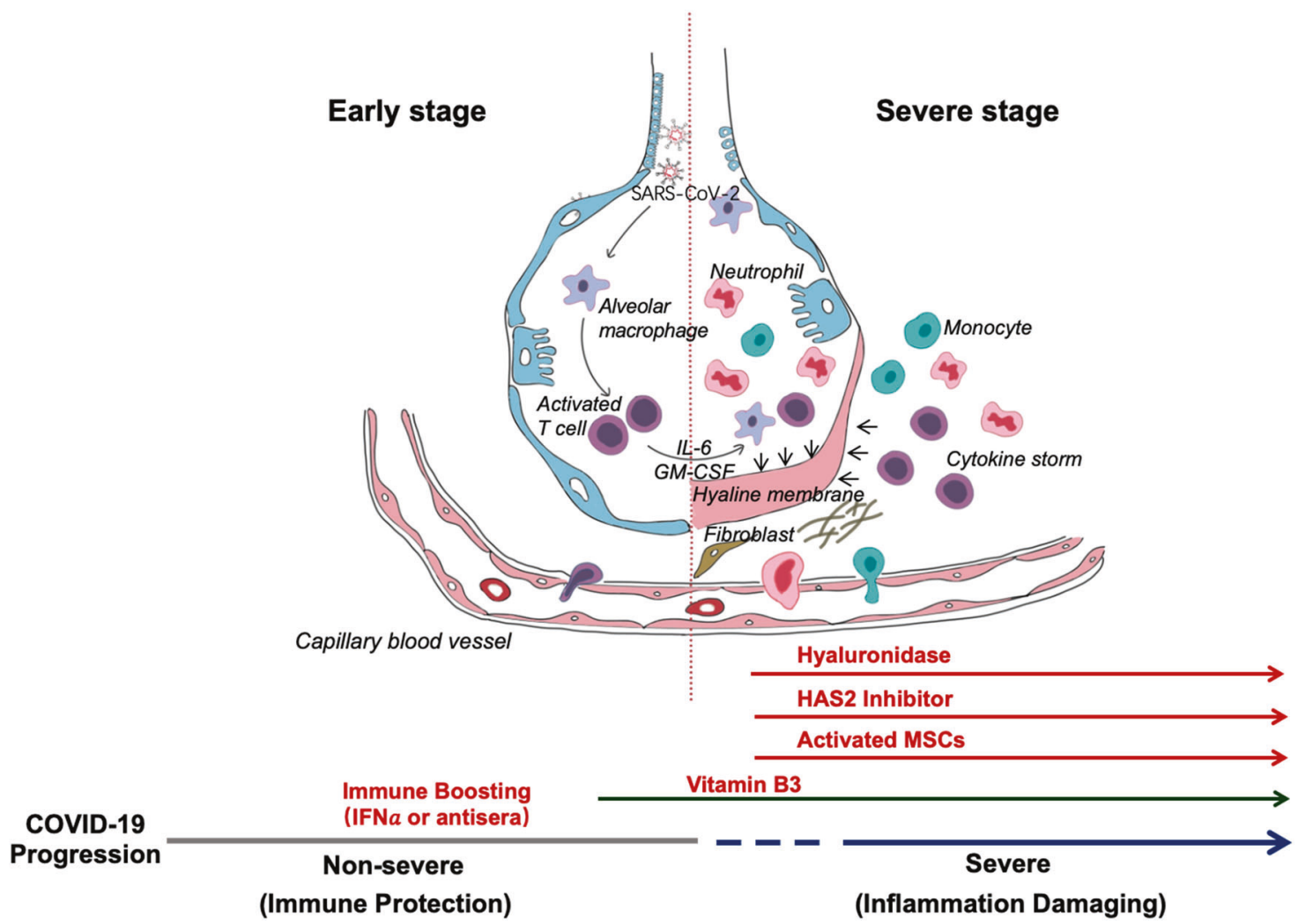

Fig. 1 Schematic representation of the progression of COVID-19 infection and potential adjuvant interventions. After an incubation period, the invading COVID-19 virus causes non-severe symptoms and elicits protective immune responses. The successful elimination of the infection relies on the health status and the HLA haplotype of the infected individual. In this period, strategies to boost immune response can be applied. If the general health status and the HLA haplotype of the infected individual do not eliminate the virus, the patient then enters the severe stage, when strong damaging inflammatory response occurs, especially in the lungs. At this stage, inhibition of hyaluronan synthase and elimination of hyaluronan can be prescribed. Cytokine activated mesenchymal stem cells can be used to block inflammation and promote tissue reparation. Vitamin B3 can be given to patients starting to have lung CT image abnormalities. strong inducers of HA-synthase-2 (HAS2) in CD31 endothelium, $\mathrm{EpCAM}^{+}$lung alveolar epithelial cells, and fibroblasts [13]. Importantly, HA has the ability to absorb water up to 1000 times its molecular weight. Therefore, reducing the presence or inhibiting the production of HA holds a great promise in helping COVID-19 patients breathe. Doctors can simply provide patients medical grade hyaluronidase to reduce the accumulation of HA and thus to clear the jelly in the lung. In animal models, influenzainduced breathing difficulties can be relieved by intranasal administration of hyaluronidase. Doctors can also use a clinically approved bile therapy drug, Hymecromone (4Methylumbelliferone, 4-MU), an inhibitor of HAS2 [14]. LPS-induced lung inflammation can be relieved by 4-MU. 4-MU or its chemical derivatives exist widely in various herbs used in traditional Chinese medicine, which may explain the observed effectiveness of combined herbal medicine in some patients.

Overall, this synopsis is based on some clinical common sense. We propose some simple, but largely ignored, approaches to the treatment of COVID-19 patients (Fig. 1).
We believe that the two-phase division is very important: the first immune defense-based protective phase and the second inflammation-driven damaging phase. Doctors should try to boost immune responses during the first, while suppressing it in the second phase. Since Vitamin B3 is highly lung protective, it should be used as soon as coughing begins. When breathing difficulty becomes apparent, hyaluronidase can be used intratracheally and at the same time 4-MU can be given to inhibit HAS2. Of course, HLA typing will provide susceptibility information for strategizing prevention, treatment, vaccination, and clinical approaches. We hope that some of the above ideas can be employed to help combat this deadly contagious disease of increasing incidence around the world.

Acknowledgements The work has been partially supported by grants from the National Key R\&D Program of China (2018YFA0107500), the Scientific Innovation Project of the Chinese Academy of Sciences (XDA16020403), Suzhou 2020 Emergency Innovation Funding on COVID-19 Infection, the National Natural Science Foundation of China (81530043, 81861138015, 31771641 and 81571612), PANDORA-ID-NET (to GI), the European \& Developing Countries Clinical Trials Partner-ship, supported under Horizon 2020 to GI), Italian Ministry of Health (RC, 1 to MP and GI). 


\section{Compliance with ethical standards}

Conflict of interest The authors declare that they have no relevant conflict of interest.

Publisher's note Springer Nature remains neutral with regard to jurisdictional claims in published maps and institutional affiliations.

\section{References}

1. Guan WJ, Ni ZY, Hu Y, Liang WH, Ou CQ, He JX, et al. Clinical Characteristics of Coronavirus Disease 2019 in China. The New England journal of medicine. 2020. https://doi.org/10.1056/ NEJMoa2002032.

2. Wang D, Hu B, Hu C, Zhu F, Liu X, Zhang J, et al. Clinical Characteristics of 138 Hospitalized Patients With 2019 Novel Coronavirus-Infected Pneumonia in Wuhan, China. Jama. 2020. https://doi.org/10.1001/jama.2020.1585.

3. Rothe C, Schunk M, Sothmann P, Bretzel G, Froeschl G, Wallrauch C, et al. Transmission of 2019-nCoV Infection from an Asymptomatic Contact in Germany. The New England journal of medicine. 2020;382:970-1. https://doi.org/10.1056/NEJMc2001468.

4. Xu Z, Shi L, Wang Y, Zhang J, Huang L, Zhang C, et al. Pathological findings of COVID-19 associated with acute respiratory distress syndrome. The Lancet Respiratory medicine. 2020. https://doi.org/10.1016/S2213-2600(20)30076-X.

5. Wang Y, Chen X, Cao W, Shi Y. Plasticity of mesenchymal stem cells in immunomodulation: pathological and therapeutic implications. Nat Immunol. 2014;15:1009-16. https://doi.org/10.1038/ ni.3002.

6. Wang G, Cao K, Liu K, Xue Y, Roberts AI, Li F, et al. Kynurenic acid, an IDO metabolite, controls TSG-6-mediated immunosuppression of human mesenchymal stem cells. Cell death and differentiation. 2018;25:1209-23. https://doi.org/10.1038/s41418017-0006-2.

7. Nagai A, Matsumiya H, Hayashi M, Yasui S, Okamoto H, Konno $\mathrm{K}$. Effects of nicotinamide and niacin on bleomycin-induced acute injury and subsequent fibrosis in hamster lungs. Experimental lung research. 1994;20:263-81. https://doi.org/10.3109/ 01902149409064387.

8. Blackwell JM, Jamieson SE, Burgner D. HLA and infectious diseases. Clin Microbiol Rev. 2009;22:370-85. https://doi.org/10. 1128/CMR.00048-08.

9. Matzaraki V, Kumar V, Wijmenga C, Zhernakova A. The MHC locus and genetic susceptibility to autoimmune and infectious diseases. Genome Biol. 2017;18:76. https://doi.org/10.1186/ s13059-017-1207-1.

10. Dutta M, Dutta P, Medhi S, Borkakoty B, Biswas D. Polymorphism of HLA class I and class II alleles in influenza A (H1N1)pdm09 virus infected population of Assam, Northeast India. J Med Virol. 2018;90:854-60. https://doi.org/10.1002/jmv. 25018.

11. MacLaren G, Fisher D, Brodie D. Preparing for the most critically Ill patients with COVID-19: the potential role of extracorporeal membrane oxygenation. JAMA. 2020. https://doi.org/10.1001/ja ma.2020.2342.

12. Hallgren R, Samuelsson T, Laurent TC, Modig J. Accumulation of hyaluronan (hyaluronic acid) in the lung in adult respiratory distress syndrome. Am Rev Respir Dis. 1989;139:682-7. https:// doi.org/10.1164/ajrccm/139.3.682.

13. Bell TJ, B O, Morgan DJ, Salek-Ardakani S, Jagger C, Fujimori $\mathrm{T}$, et al. Defective lung function following influenza virus is due to prolonged, reversible hyaluronan synthesis. Matrix Biol. 2018;80:14-28.

14. Collum SD, Chen NY, Hernandez AM, Hanmandlu A, Sweeney $\mathrm{H}$, Mertens TCJ, et al. Inhibition of hyaluronan synthesis attenuates pulmonary hypertension associated with lung fibrosis. British journal of pharmacology. 2017;174:3 284-301. https://doi.org/10. 1111/bph.13947. 